\title{
Rational psychotherapy of neuroses: dynamic changes in psychological response of men and women
}

\author{
Irina Skirtach ${ }^{1,2, *}$, Ekaterina Denisova ${ }^{1}$, and Victoria Korkhova $^{1}$ \\ ${ }^{1}$ Don State Technical University, 344003, Gagarin Sq., 1, Rostov-on-Don, Russia \\ ${ }^{2}$ Southern Federal University, 344006, Bolshaya Sadovaya str., 105/42, Rostov-on-Don, Russia
}

\begin{abstract}
The paper presents the results of a study of dynamic changes in the severity of depression, it's somatic manifestations, basic beliefs and dysfunctional attitudes in the course of treatment which included rational psychotherapy. The study sample consisted of 48 men and women with different forms of prediagnosed neurotic disorder aged 25-44 years $(\mathrm{M}=$ 32.6, $\mathrm{SD}=5.3(43.7 \%$ men)). Methods: Beck Depression Inventory (BDI, N.V. Tarabrina, 2001), Beck Anxiety Inventory (BAI, N.V. Tarabrina, 2001), Janoff-Bulman The World Assumptions Questionnaire (M.A. Padun, A.V. Kotelnikova, 2007), A. Beck, A. Weissman Dysfunctional Attitudes Scale (DAS, M.L. Zakharova, 2013). Statistical analysis included Kruskal-Wallis H-test and one-way ANOVA with post-hoc analysis. As a result of the study, it was possible to identify gender differences in the dynamics of cognitive-affective manifestations of depression, somatic manifestations of depression and dysfunctional relationships. It has been found that women demonstrate greater progress in stabilizing their emotional state and developing the skills to recognize their dysfunctional thoughts. At the same time, men are distinguished by a more pronounced dynamics of change in beliefs about their own value, the ability to manage events and luck.
\end{abstract}

\section{Introduction}

In recent decades, neurotic disorders have had a high prevalence in the population (official statistics for individual regions of Russian Federation shows that a confirmed diagnosis rate is 15.8 to 21.8 per 1000 people). The number of works focused to the clinical and psychological aspects and therapy for neurotic disorders has been increasing accordingly [1-5]. However, the problem of neurotic disorders remains relevant, attracting the attention of a wide range of researchers, including psychologists.

Among all mental diseases, neurotic spectrum disorders account for $20-25 \%$ [6]. In addition, the role of the psychological factor in the etiopathogenesis of neurotic disorders and its effects on the specificity of neurotic conflicts, the experience of traumatic situations and the clinical picture of the disease determine the need for further research in this area [7]. Previous studies have shown that the use of rational psychotherapy in the correction of

\footnotetext{
${ }^{1}$ Corresponding author: limpopo-is@yandex.ru
} 
indicators of the emotional states and cognitive biases (attitudes) of male and female patients is very promising. The study of the gender differences can make it possible to clarify the picture of psychopathological manifestations and create a basis for developing a therapy programs for eliminating maladaptive pathological reactions and behavioral patterns.

A neurosis is a disease caused by the impact of traumatic factors or events. It is characterized by the reversibility. Its manifestations include emotional-affective symptoms, disturbance in somato-vegetative functions and mental exhaustion. A patients suffering from neuroses are aware of the fact of their disease and remain critical of their condition or external circumstances [8-10].

Distorted, false cognitions serve as the basis for this disorder. They cause the formation of dysfunctional ideas, as a result of which the patient develops inappropriate emotional reactions [11-12]. With this disorder, a person can to experience a wide range of affective symptoms: depression, asthenia, decreased volitional control, fears and phobias, anhedonia, melancholy and anxiety. The forms of neurotic disorders are usually classified based on the severity of these symptoms [13].

The cognitive approach considers neurotic disorder as a result of the formed maladaptive cognitive schemes. These schemes organize experience, perception, judgment, feelings and behavior of the individual. As a result, the patient develops a behavioral maladaptation dictated by the accumulation of automatic thoughts, systematic cognitive errors and perversions of fundamental emotions. Errors in the perception of the reality, which are characterized by unrealism, inconsistency, irrationality, lead to a distortion of perception and assessment of oneself and the environment. As a result, the systemic components of interpersonal relationships (emotional, behavioral) are deformed, which becomes a trigger factor for the emergence of neurotic and psychosomatic disorders [13].

The most widely recognized clinical treatment for neurotic disorders is the administration of pharmacological agents (drugs). In some cases, drug therapy is carried out in combination with physiotherapy, acupuncture and herbal medicine, as well as psychotherapy. Person-oriented (reconstructive) therapy, hypnotic suggestion (while awake, hypnotic sleep, auto-training), behavioral therapy and art-therapy can be mentioned among the most commonly used psychotherapeutic approaches in the treatment of neuroses [8,14-16]. Rational psychotherapy as a kind of reconstructive psychotherapy involves conversations with the patient, during which the circumstances of the onset of the disease are worked out. It also includes an explanation of the reversibility of the resulting violations, the correction of the patients' ideas about the disturbing (trigger) events, and the removal of fixation on pathological symptoms. The therapeutic effect is achieved through the information, which, interacting with the patients' maladaptive schemes, subject them to processing and critical assessment [17]. Rational psychotherapy is recommended in cases where differential verbal interactions are possible, and the patient is characterized by a sufficient level of mental flexibility, has good motivation and is ready to take an active part in understanding the reasons and interconnection of the disease with the realities of life and experiences, as well as the inadequacy of his/her own reactions. The expected positive result of such therapy is the development of new views and attitudes towards things, a new ways of assessment and dealing with traumatic events, new attitudes towards the future [17, $18]$.

Previous studies conducted on patients with neurotic disorders mainly cover a wide range of psychological states and traits, but the analysis is usually limited to identifying differences in the psychological characteristics of patients with different forms of neurotic disorders [19-21]. But little attention has been paid to gender differences in etiology, manifestations and severity of psychological states and symptoms of the neurotic spectrum [7, 22-25]. So the study of Isurina G.L. showed that men are characterized by a higher level 
of severity of symptoms, while women are characterized by a higher severity of neurotic personality traits [7]. In the study of Medvedev N.N. and Babin S.M. devoted to the gender differences in compensation of self-attitude at different levels of neurotic states, it is shown that women with neurosis extremely externalize their own self-esteem, expecting a positive assessment from others. It is also shown that they oppose themselves to others and have a strong distortion of self-criticism. Decompensation of cognitive attempts at self-regulation occurs, while low self-esteem is under the influence of affective compensatory mechanisms. For men with neurosis the main vector of compensation for self-attitude is the idealization of their own Self. They seek confirmation of this from others, which results in an increased dependence in interpersonal relationships. This is aggravated by general passive life attitudes and fixation on maladaptive patterns of behavior, which reduces the possibility of spontaneous recovery (or even professionally helped recovery) due to the fact that they refuse to accept and exercise new behavioral patterns [24]. Therefore, prior investigations of gender differences in the manifestations and severity of neurotic symptoms as well as compensatory mechanisms suggest that there may be differences in how they will respond to treatment with rational psychotherapy.

\section{Methods}

The sample consisted of 48 people ( 27 women, 21 men). The study was conducted at the State Budgetary Healthcare Institution "Specialized Psychoneurological Hospital" of the Ministry of Health of the Krasnodar Region. All participants were diagnosed with a neurotic disorder of the nosological blocks F40-F48 Neurotic, stress-related and somatoform disorders (according to ICD-10 Version:2019).

The age of the subjects ranged from 25 to 44 years $(\mathrm{M}=32.6, \mathrm{SD}=5.3(43.7 \% \mathrm{men}))$ that corresponds to a young age according to WHO age grouping of population.

The sample was divided into control and experimental groups of equal size. All patients during the study were treated in inpatient conditions using a standard drug therapy regimen. A course of rational psychotherapy (14 individual sessions lasting 1.5 hours) was introduced into the treatment program for the experimental group. In all cases patients' consent was obtained.

As a hypothesis of the study, we suggested that there are differences in the response to rational psychotherapy in men and women with neurotic disorders. These differences were measured through the level of depression, anxiety, basic beliefs, and the severity of dysfunctional attitudes.

The following psychological tests were used to measure these characteristics: Beck Depression Inventory (BDI, N.V. Tarabrina, 2001), Beck Anxiety Inventory (BAI, N.V. Tarabrina, 2001), Janoff-Bulman The World Assumptions Questionnaire (M.A. Padun, A.V. Kotelnikova, 2007), A. Beck, A. Weissman Dysfunctional Attitudes Scale (DAS, M.L. Zakharova, 2013). Russian-language versions of these questionnaires had the highest validity and allowed us to study the somatic, cognitive and emotional symptoms of neurotic anxiety and depression.

Statistical analysis included Kruskal-Wallis test and one-way ANOVA with post-hoc analysis. These data analysis techniques properly employ the pooled variance with binary factor in multiple comparison on non-equivalent groups.

The interviewees with psychological testing were carried out at the beginning of the study upon patients' admission to the hospital. That allowed us to measure the severity of somatic, cognitive and emotional symptoms before patients received any treatment. Same characteristics was re-tested after the they underwent a full standard course of treatment and the course of rational psychotherapy (in the experimental group). 


\section{Results}

The results of the analysis of differences in the indicators of the emotional states, basic beliefs and attitudes of neurotic patients before and after undergoing treatment in a hospital, taking into account the types of neurotic disorders, are described in detail in our previous paper [26]; in this work, we consider it necessary to focus on the gender specificity of the dynamics of the studied indicators.

The effect of including rational psychotherapy in the treatment process was evaluated. Comparison of the dynamics of the studied indicators of men and women in the control and experimental groups was carried out using the Kruskal-Wallis test. We were able to establish the presence of a statistically significant effect of the "rational therapy" factor on the dynamic changes of the severity of cognitive-affective manifestations of depression, somatic symptoms of depression, basic beliefs, dysfunctional attitudes $(p \leq 0.05)$.

Statistical analysis showed that in the experimental group (participating in psychotherapy) the dynamics of the level of depression, the severity of dysfunctional relationships, the nature of some basic beliefs of female patients with neurotic disorders differs from the results of male patients. This indicates the presence of gender differences in the change in the psychological response of patients (Table 1).

Table 1. Comparison of the dynamics of the studied indicators in men and women groups using oneway ANOVA

\begin{tabular}{|l|l|l|}
\hline Indicators (variables) & $\mathbf{F}$ & $\mathbf{p}$ \\
\hline Changes in cognitive-affective manifestations of depression & $8,48008^{*}$ & 0,004079 \\
\hline Changes in somatic manifestations of depression & $12,65967^{*}$ & 0,003226 \\
\hline Changes in anxiety levels & 2,16135 & 0,148636 \\
\hline $\begin{array}{l}\text { Changes in the severity of dysfunctional beliefs on the scale of } \\
\text { "benevolence of the world" }\end{array}$ & 0,89742 & 0,348648 \\
\hline $\begin{array}{l}\text { Changes in the severity of dysfunctional beliefs on a scale of } \\
\text { "justice" }\end{array}$ & 0,58649 & 0,447867 \\
\hline $\begin{array}{l}\text { Changes in the severity of dysfunctional beliefs on "the self- } \\
\text { value and self-significance" }\end{array}$ & $14,78169^{*}$ & 0,000385 \\
\hline $\begin{array}{l}\text { Changes in the severity of dysfunctional beliefs on the "luck" } \\
\text { scale }\end{array}$ & 0,01931 & 0,890112 \\
\hline Changes in the severity of dysfunctional beliefs about "control" & $40,24161^{*}$ & 0,0000001 \\
\hline Changes in the severity of dysfunctional attitudes & $10,95860^{*}$ & 0,001866 \\
\hline
\end{tabular}

Notes: * - statistically significant differences

Post-hoc analysis showed that women significantly differ from men in the dynamics of cognitive-affective manifestations of depression, while the effect is greater in the female group ( $\mathrm{p} \leq 0.05$; Effect size $\mathrm{d}_{\text {women }}=-8,500$; Effect size $\mathrm{d}_{\text {men }}=-5,533$; $\mathrm{MS}=19.323, \mathrm{df}=$ 22.00). In people with neurotic disorders, the adaptive capabilities of the psyche are reduced, they need a longer time to master the changes that have arisen ( 6 months - 1 year). Rational psychotherapy is structured in such a way that it helps patients to quicker realization of their problems so they would be able to change their attitude towards them. It is aimed at depressive dysfunctional attitudes, reinforces alternative thinking, through new ways of thinking, builds new patterns of behavior (ones of an adaptive nature). The emotional background changes (positively), therefore, the manifestations of depression decrease [17].

Post-hoc analysis showed that women significantly differ from men in the dynamics of somatic symptoms of depression, while the effect is greater in women $(p \leq 0.05$; Effect size $\mathrm{d}_{\text {women }}=-5.259$; Effect size $\mathrm{d}_{\text {men }}=-2.476$; MS $\left.=20.457, \mathrm{df}=46.00\right)$. In the course of 
rational psychotherapy, a patient learns the laws of formal logic and uses them to find errors in thinking. As a result, a patient comes to an adequate attitude to his illness. Through the efforts of your own thinking, the level of fear for your health is reduced. Thus, the somatic symptoms of the disorder (sleep disturbances, loss of appetite, inhibition of libido) decrease [27].

Significant differences in the nature of basic beliefs were identified for "beliefs about self-value and self-significance" $(\mathrm{p} \leq 0.05$; Effect size $\mathrm{d}$ women $=1.173$; Effect size $\mathrm{d}$ men $=$ 2.320; $\mathrm{MS}=2.8240$, df $=54.00)$, with a larger effect size for men. The studied subjects belong to the young age group, which means that they may lack the consistent image of themselves. However, at this age, ideas about self-realization are already well-formed, there is a need to unleash their potential in professional activity and by building a family. Along with this, patients may have a problem of decreasing physical strength and may feel attractive (especially for women), which is severe a blow to self-esteem and self-concept. The listed negative personality changes associated with neurotic disorders require psychological interventions and, according to the results obtained, rational psychotherapy will be effective in correction of the beliefs on "the self-value and self-significance". The greater effect was noted in men. Since the techniques of rational psychotherapy are aimed at restoring the basic belief about the value and significance of one's own self results in positive changes. In women, a reassessment of values, a critical revision of their self-image occurs slower. This may explain not such pronounced response to therapy aimed at restoring the positivity of the self-image.

Post-hoc analysis showed significant differences when comparing the results of men and women on the dynamics of indicators of the basic beliefs about "control" $(p \leq 0.05$; Effect size $\mathrm{d}_{\text {women }}=0.4733$; Effect size $\mathrm{d}_{\text {men }}=1.8958$; $\mathrm{MS}=14.531$, $\mathrm{df}=116.00$ ). This scale reflects the belief of the individuals that they can control the events taking place in their lives, and also can actively change the situation in their favor. Based on the data obtained, we can assume that women meet therapeutic interventions about this cognitive distortion with greater resistance than men. The cognitive scheme of women is less flexible, due to the greater emotional coloring of painful experiences, which is associated with a neurotic disorder; therefore, they with great difficulty abandon these dysfunctional beliefs.

Significant differences in the changes in the severity of dysfunctional beliefs on the "luck" scale were also found ( $\mathrm{p} \leq 0.05$; Effect size $\mathrm{d}$ wives $=1.2222$; Effect size $\mathrm{d}$ husband = 2.5833; $\mathrm{MS}=3.7941$, df $=58,000$ ). This group of basic beliefs reflects the idea of an individuals about themselves in terms of "good luck - bad luck". When assessing themselves, men often pay the most attention to intellectual and volitional qualities: responsibility, dedication, perseverance, self-control, creativity. In general, they are more result-oriented, and therefore more demanding of themselves. These qualities are negatively sharpened in neurotic spectrum disorders, which reduces the beliefs in good luck and a successful result. Rational psychotherapy aims to increase self-confidence and selfconfidence. As a result, self-esteem, an understanding of one's abilities, qualities, merits, skills and strengths increases - therefore, self-respect, faith in success and luck grows. According to the results obtained, the response to rational psychotherapy is more pronounced in men.

We also managed to establish that there are significant differences in the dynamics of the severity of the dysfunctional attitudes in men and women undergoing rational psychotherapy. ( $\mathrm{p} \leq 0.05$; Effect size $\mathrm{d}_{\text {women }}=-12,000$; Effect size $\mathrm{d}$ men $=-9,500, \mathrm{MS}=$ 340.63 , $\mathrm{df}=46,000)$. A more pronounced positive effect in relation to the correction of the dysfunctional attitudes is shown in women. Irrational thoughts may be a consequence and also a cause of mental disorder. These are maladaptive thoughts can provoke negative emotions, various physiological problems, and set off a neurotic behavior. While working with a psychotherapist, patients develop the skill of recognizing their dysfunctional 
thoughts, replacing them with more adaptive and rational ones. Rational psychotherapy is argumentation for proving or refuting beliefs associated with the patient's idea of the essence of certain issues related to the disease, this is an intellectual struggle, during which the logical inconsistency of patient's thinking and conclusions are being corrected.

\section{Conclusions}

The described dynamic changes in psychological response of patients participating in the treatment program, which included rational psychotherapy, has clear signs of positive effects of including individual psychotherapy in the standard drug treatment. In comparison with the control group, there were some improvements in terms of correcting the level of depression, anxiety, the nature of basic beliefs and the severity of the dysfunctional attitudes both men and women.

The observed changes are achieved by correcting the informational meaning of stimuli that carry a pathogenic charge for the psyche. Patients become able to look at problems (including their disease) and the world around them more objectively. Their attitude towards the events that led to the disorder acquires adequacy. What previously caused painful experiences is now losing its relevance and significance. Patients, step by step, through the efforts of their own mind, comes to see new reactions and behavior patterns. This allows you to reduce health-related concerns, get rid of obsessive pathogenic thoughts, increase general awareness, and increase self-esteem. This naturally leads to an increase in the energy potential and stabilization of the emotional sphere and opens up internal psychological resources that can be used not for destruction, but for growth.

Analysis of gender differences in the dynamics of the severity of the characteristics of the cognitive and emotional spheres in the experimental group showed that rational psychotherapy has a greater effect on women in cognitive-affective and somatic manifestations of depression, as well as in correction of dysfunctional beliefs and attitudes. Despite the fact that we did not observe differences in changes in the level of anxiety in men and women, the data obtained may indicate different mechanisms that men and women use to cope with it. Women are prone to greater intensity of emotional reactions. They are traditionally distinguished by a tendency to greater somatization. Whereas male coping strategies gravitate towards cognitive restrictive mechanisms. This is possibly imposed by a number of gender stereotypes that require men to have greater mental stability and selfconfidence. This gives rise to a cognitive fixation on the malignity of neurotic manifestations of the disease for the men's role in the social, physiological and psychological aspects. The reported results confirm the hypothesis. The presence of a greater response to psychotherapy in men is established while working on basic beliefs associated with beliefs about self-worth, the ability to manage events and luck.

We undertook this study in order to show the possibilities and limitations of using rational psychotherapy methods depending on the gender of the patient with different forms of neurotic disorder. The obtained results and conclusions can become the basis for constructing an optimal rehabilitation program and more accurately predicting the dynamics of improving the condition of patients.

\section{References}

1. J. A. Sobański, et al., Psychiatr Pol, Changes in ego strength in patients with neurotic and personality disorders treated with a short-term comprehensive psychodynamic psychotherapy, 52(1), 115-27 (2018) doi: 10.12740/PP/OnlineFirst/40020 
2. A. Vasileva, T. Karavaeva, S. Lyashkovskaya, Archives of Psychiatry and Psychotherapy, Typology of psychotherapeutic targets and changes in state of patients with neurotic disorders in the course of personality-oriented (reconstructive) psychotherapy, 4, 22-31 (2017) doi: 10.12740/APP/80167

3. M. Mielimąka, et al., Psychiatr. Pol, Trait and state anxiety in patients treated with intensive short-term group psychotherapy for neurotic and personality disorders, 51(6), 1165-1179 (2017) doi: 10.12740/PP/OnlineFirst/60537

4. T. A. Karavaeva, et al., S.S. Korsakov, Journal of Neurology, A Comparative Study of the Efficacy of Personality-Oriented (Reconstructive) and Cognitive-Behavioral Psychotherapy in Neurotic Anxiety Disorders With Insomnia, 118(4), 2, 60-66 (2018) doi:10.17116/jnevro20181184260.

5. F, Zheng, et al., Journal of International Medical Research, Somatic symptoms and their association with anxiety and depression in Chinese patients with cardiac neurosis, 47(10), 4920-4928 (2019) doi: 10.1177/0300060519869711

6. Y. V. Malygin, V. L. Malygin, S. A. Shamov, D. B. Tsygankov, S. S. Korsakov, Journal of Neurology and Psychiatry. Special issues. A multiple model of help-seeking behavior in patients with depressive and neurotic disorders, 119(1), 17-21 (2019) doi: 10.17116/jnevro20191191217

7. G. L. Isurina, I. V. Grandilevskaya, P. K. Trombczynski, Petersburg Psychological Journal, Gender differences in the severity of neurotization of the personal and clinical and psychological level, 21, 64-78 (2018)

8. E. L. Nikolaev, E. S. Suslova, Medical Psychology in Russia, Dynamics of scientific ideas about neuroses: from a biological model to a cultural one, 5(10) (2011)

9. V. A. Gilyarovsky, Soviet Neuropathology, Psychiatry and Psychohygiene, Key points in the problem of neuroses, 3(2-3), 74-86 (2014)

10. J. A. Sobański, et al., Psychiatr. Pol, Occurrence of selected lower urinary tract symptoms in patients of a day hospital for neurotic disorders, 50(6), 1181-1205 (2016) doi:10.12740/PP/OnlineFirst/61109

11. T. M. Lavrinovich, Bulletin of Chelyabinsk State Pedagogical University [Vestnik ChGPU]. Comparative analysis of the views on the neurosis nature in non-medical psychotherapy, 5, 80-84 (2015)

12. Babl et al., Journal of affective disorders, Comparison and change of defense mechanisms over the course of psychotherapy in patients with depression or anxiety disorder: Evidence from a randomized controlled trial, 252, 212-220 (2019)

13. N. N. Lebedeva et al., S. S. Korsakov, Journal of Neurology and Psychiatry, Neurophysiological peculiarities of cognitive activity in patients anxiety-depressive and hypochondriacal disorders, 119(3), 43-49 (2019)

14. M. A. Lebedev, S. Yu. Palatov, G. V. Kovrov, Russian Medicine Journal. Medical Review, Neuroses (clinic, dynamics, therapy), 21(3), 165-168 (2013)

15. M. Khademi, M. Hajiahmadi, M. Faramarzi, Trends in psychiatry and psychotherapy, The role of long-term psychodynamic psychotherapy in improving attachment patterns, defense styles, and alexithymia in patients with depressive/anxiety disorders, 41(1), 4350 (2019) doi: 10.1590/2237-6089-2017-0144

16. Y. V. Malygin et al., S. S. Korsakov, Journal of Neurology and Psychiatry, A multiple linear model of help-seeking behavior in patients with depressive and neurotic disorders, 119(1), 17-21 (2019) doi: 10.17116/jnevro20191191217 
17. D. Evstigneev, Bulletin of Ulyanovsk Civil Aviation Institute n.a. B.P. Bugaev, Rational psychotherapy: history of formation, 9, 106-117 (2017)

18. S. S. Odarchenko, A. I. Cheperin, Omsk Psychiatric Journal, Psychotherapy and rehabilitation of elderly patients, 1(1), 41-43 (2014)

19. M. Holubova, et al., Psychology research and behavior management, Quality of life, self-stigma, and coping strategies in patients with neurotic spectrum disorders: a crosssectional study, 12, 81 (2019)

20. Prasad, CRC Press, Biological basis and therapy of neuroses, 202 (2018)

21. N. K. Bogolepov, The problem of the examination of the work ability and diagnosis of borderline states (psychoneuroses), Neurotic reactions after emotional trauma, 9, 128 (2014)

22. J. Busfield, Macmillan International Higher Education, Men, women and madness: Understanding gender and mental disorder (2017)

23. L. Gul, S. F. Jahangir, FWU Journal of Social Sciences, The Effectiveness of Mindfulness-Based Stress Reduction Programme (MBSRP) and Sufi Meditation (SM) in the Treatment of Neurotic Anxiety among Females, 13(1) (2019)

24. N. Medvedev, S. Babin, Bulletin of Psychotherapy, Conditionality of gender differences in compensation of self-attitude at different levels of neurotic states, 68, 2946 (2018)

25. N. Tkachenko, Fundamental aspects of mental health, Systematic analysis of the pathogenesis of hysterical neurosis in women, 4, 109-114 (2016)

26. I. Skirtach et al., SHS Web of Conferences, Effects of rational psychotherapy on emotional state and cognitive attitudes of patients with neurotic disorders, 70, 09011 (2019) doi:10.1051/shsconf/20197009011 